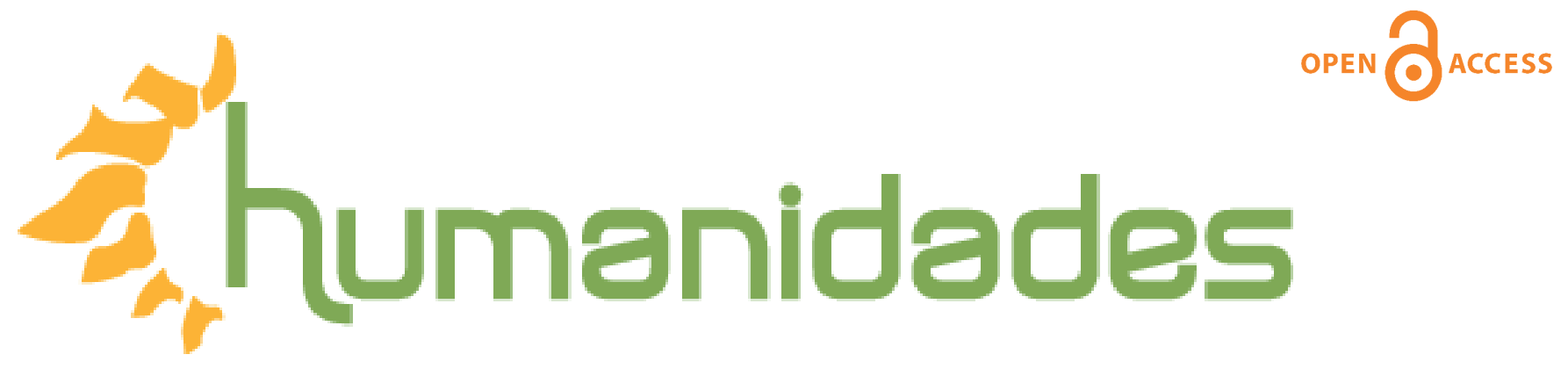

Revista de la Escuela de Estudios Generales, Universidad de Costa Rica

Julio-diciembre, 2018 •Volumen 8, número 2 • EISSN 2215-3934 •pp. 1-33

Recibido: 22-Febrero-2018 Aceptado:30-Abril-2018

\title{
Aprendiendo a lo largo de la ciudad: la carrera de los estudiantes de actuación en Buenos Aires
}

DOI: http://dx.doi.org/10.15517/h.v8i2.33339

\section{Lic. Santiago Battezzati}

Licenciado en Ciencias Políticas y de Gobierno. Instituto de Altos Estudios Sociales (IDAES), Universidad Nacional de San Martín, Argentina.

Correo electrónico: santiagobattezzati@gmail.com

Todos los derechos reservados. Universidad de Costa Rica. Esta revista se encuentra licenciada con Creative Commons. Reconocimiento-NoComercial-SinObraDerivada 3.0 Costa Rica. Correo electrónico: humanidades@ucr.ac.cr / Sitio web: http://revistas.ucr.ac.cr/index.php/ humanidades 


\title{
Aprendiendo a lo largo de la ciudad: la carrera de los estudiantes de actuación en Buenos Aires
}

\section{Resumen}

Varias investigaciones han descrito la circulación entre estudios o escuelas como parte de la práctica y o aprendizaje de ciertas disciplinas en ciudades. En este trabajo intento delimitar ciertas formas específicas de circulación, tal como he podido reconstruirlas a partir de una investigación etnográfica entre estudiantes de actuación en Buenos Aires. Reconstruyendo estas trayectorias a partir del concepto beckeriano de carreras secuenciales, destaco la necesidad de prestar atención a la temporalidad de estas carreras -encontrando diferentes modos de circular o no en diferentes instancias de estas carreras- de forma complementaria a ciertos estudios que han destacado la importancia de la espacialidad y el espacio urbano para dar cuenta de dichas circulaciones.
Palabras claves: Teatro, Circuito, Aprendizaje

\section{Learning Along the City: the Career of Acting Stu- dents in Buenos Aires}

\begin{abstract}
Some researchs have described the circulation between different schools as part of the learning process of certain disciplines in cities. In this work I describe some specific ways of circulation between theater students in Buenos Aires, the issue of my current etnographic research. Drawing on the beckerian concept of sequential carrees, I focus on the temporality of these carreers -showing there are different ways of circulating in different moments of the learning process-. I thus try to complement others studies that have focused on spatiality and urban space as the main feuture to describe similar phenomena.
\end{abstract}

Keywords: Performance, Circuit, Learning 
Los estudios y escuelas de actuación en la Ciudad de Buenos Aires son muchos. Si bien no existen estadísticas -ya que muchas de estas escuelas funcionan de manera informal en casas viejas refaccionadas como escuelas y pequeñas salas y/o centros culturales-, el sitio Alternativa Teatral (www. alternativateatral.com) señala 418 "espacios" donde se realizaron espectáculos en el off 1. Tal como pude constatar a lo largo de mi trabajo de campo,2 la gran mayoría de las salas - de no más de cien butacas- que funcionan en estos "espacios" son también escuelas o estudios donde uno o varios maestros dan clases de actuación. De hecho, son las clases y no las obras las que permiten la subsistencia económica de estas salas.

En este contexto, y dado que la formación de los estudiantes de actuación suele darse en una circulación entre varios maestros y escuelas a lo largo de los años, las combinaciones y los recorridos posibles son enormes -sin contar el hecho de que los estudiantes de actuación también suelen circular por clases de otras disciplinas como danza, estudio de instrumentos, o terapias o prácticas nueva era, entre muchos otros-. Acotando la formación al entrenamiento en estudios de actuación, podemos pensar que esta va desde que un estudiante se anota en un primer curso de actuación hasta que deja de entrenar -lo que en muchas ocasiones sólo sucede cuando el estudiante no tiene tiempo para seguir entrenando, por estar ensayando obras o participando en otros proyectos-. Este trabajo buscará dar cuenta de algunos de esos recorridos: los que acontecen entre estudiantes que en algún momento de su formación fueron cautivados por un maestro por un período largo de tiempo.

Varios estudios han señalado la circulación entre estudios y/o maestros como un rasgo central del aprendizaje y/o la realización de ciertas prácticas, como Magnani (1999) para el circuito neoesotérico en San Pablo, Carozzi (2000) para el circuito alternativo en Buenos Aires, Osswald (2015) para la danza independiente en Buenos Aires, del Mármol (2016) para el aprendizaje de teatro en La Plata, y Saez (2017) para la danza contemporánea y el circo en La Plata. Particularmente influyente ha sido el estudio seminal de Magnani (2002, 2006).

Este antropólogo desarrolla un enfoque que pone en diálogo los estudios ur-

\section{Introducción}


banos con los estudios sobre juventud y analiza el modo en que los jóvenes se apropian de los espacios urbanos y recorren la ciudad (Magnani, 2006). Magnani (2002) también desarrolla varios conceptos -como pedaço, mancha, trajeto y circuito - que permiten entender a diferentes grupos de jóvenes en su relación con el espacio urbano que ocupan y transitan. Particularmente interesante es la noción de circuito que Magnani define como

el ejercicio de una práctica o la oferta de determinados servicios por medio de establecimientos, y espacios que no mantienen entre sí una relación de contigüidad espacial, siendo reconocido en su conjunto por los usuarios habituales: por ejemplo, el circuito gay, el circuito de cines de arte, o el circuito neoesotérico, de los salones de danza y shows negros, del pueblo de santo, de los anticuarios, de los clubbers, entre tantos otros (Magnani, 2002: 23-24).

Por otra parte, si bien la circulación como fenómeno ha sido referida para diversas prácticas y disciplinas, menos han sido los estudios que se han encargado de delimitar los modos particulares y acotados en que se da esa circulación. Uno de los pocos trabajos que ha buscado indagar, de manera sistemática, los límites de una cierta circulación, es el de Frigerio (2012). Este antropólogo se pregunta dónde se detiene el sincretismo y la reapropiación en clave new age de prácticas y creencias religiosas. A contramano de lo que ve como una tendencia académica a exaltar la inagotable capacidad sincrética del new age, Frigerio sostiene que hay ciertas religiosidades como el pentecostalismo, la devoción por los santos populares y las religiones afroamericanas, que no suelen formar parte de este sincretismo. De modo análogo a lo señalado por Frigerio, en ciertos períodos de su formación, algunos estudiantes de actuación no circulan por cualquier escuela de teatro; en cambio, acotan su recorrido entre estudios de maestros que pertenecen a un mismo estilo de actuación y que tienen en común su pertenencia a un mismo linaje. Al analizar el recorrido por un circuito como parte de una carrera secuencial más amplia (Becker, 2012), en este trabajo me propongo ver los diferentes modos en que algunos estudiantes circulan - $\mathrm{O}$ no- durante su formación, así como el modo en que se llega a circular en un circuito. Como implican por definición las carreras secuenciales $-\mathrm{y}$ este es su principal 


\section{Aprendiendo a lo largo de la ciudad...}

interés heurístico- los diferentes momentos suponen una forma distinta de orientar la acción. Tal como la fui reconstruyendo a partir de entrevistas, esta carrera suele tener cuatro momentos.

1. Los curiosos comienzan sus estudios de actuación anotándose en un curso ya sea en la escuela secundaria, en algún centro cultural del barrio, o por recomendación de un conocido o amigo que hace teatro. En ese curso de actuación escuchan nombrar a otros maestros. También van a ver obras, donde descubren actores o directores que también son maestros. Los estudiantes deciden estudiar con algunos de ellos y así van pasando de un maestro a otro, o estudian con más de uno a la vez.

2. Los estudiantes encuentran un maestro que los cautiva profundamente y estudian con él durante un período prolongado -en general de dos a cuatro años, por lo menos-. Ese maestro tiene una influencia determinante en la comprensión de estos estudiantes sobre la actuación, el modo en que empiezan a mirar, pensar, sentir, gozar, hablar, hacer y querer hacer teatro.

3. Los estudiantes continúan estudiando con otros maestros que pertenecen al mismo circuito -que tiene en común la enseñanza de un mismo estilo de actuación y la pertenencia a un mismo linaje- que el maestro que los cautivó en el momento anterior.

4. Después de un largo período de formación en circulación por estudios de un mismo circuito, algunos estudiantes empiezan a elaborar de manera propia algunas de las enseñanzas de sus maestros.Esto suele darse mientras siguen circulando -aprendiendo o realizando otras disciplinas, por otros roles ligados al teatro (como la dirección o ser maestro) o, en esta instancia, por maestros de teatro de otros estilos de actuación-.

Como ya puede haber quedado claro, aquellos estudiantes que en el tercer momento circulan por maestros de un mismo linaje tienen algo en común: han atravesado un período largo en el que fueron cautivados por un maestro -en el segundo momento-, que ha tenido una influencia determinante en el modo en que ellos conciben la actuación, es decir, en cómo hablan, piensan, sienten, miran, gozan, hacen y quieren hacer teatro. Durante mi trabajo de 
campo conocí, escuché, conversé y entrevisté a algunos estudiantes que, formándose en los más diversos estilos de actuación, habían atravesado un período en el que habían sido cautivados por un maestro.

A lo largo de este trabajo, me centro solo en las carreras de los estudiantes que han atravesado un período en el que han sido cautivado por un maestro perteneciente al circuito realista (que enseñan un estilo de actuación stanislavskiano o del circuito del teatro de estados. Brevemente, el estilo de actuación stanislavskiano se basa en las enseñanzas de Konstantin Stanislavski y busca una "verdad escénica" similar a la de la vida cotidiana. Para ello, entrena un actor capaz de compenetrarse profundamente con su personaje y sentir en escena como este siente. En las clases de este estilo de actuación, se suele dedicar bastante tiempo a componer escenas tomadas de obras de teatro. Tal como se enseña en este estilo, los estudiantes tienden a partir del análisis de la obra de teatro para comprender el personaje que van a componer. A continuación, desarrollan ciertas estrategias para entrar en el "estado" emocional que les permita actuar de un modo acorde al personaje que tienen que interpretar. Por otra parte, el estilo de actuación del teatro de estados, busca un tipo de actuación extracotidiana -en términos del movimiento y la palabra-. Este teatro entrena actores que tienden al histrionismo, capaces de articular durante la actuación estados diversos - de allí la importancia de la s final de "estados"-, por lo que no entrena tanto la compenetración emocional del actor en su personaje como una cierta economía extracotidiana de la palabra y el movimiento. Este estilo de actuación rechaza la preexistencia y la preeminencia de la palabra escrita por sobre el momento de actuación, y suele componer escenas a partir de un cierto tipo de improvisación -en lugar de a partir de obras escritas-.

En cuanto a la carrera que acabo de describir, cabe aclarar que se trata de una descripción que condensa una serie de variaciones de las que trataré de dar cuenta, y no todos los estudiantes pasan por los cuatro momentos de esta carrera. Algunos quedan cautivados por el primer maestro con el que empiezan a estudiar, y por lo tanto, el primer momento no se da. En otros casos los estudiantes se quedan estudiando durante muchos años con el maestro que los cautivó profundamente durante el segundo momento y ya no continúancirculando por otros maestros del circuito, por lo que el tercer 
momento no se da.

A continuación, describo en profundidad los cuatro momentos de esta carrera.

\section{La carrera de los estudiantes de actuación}

\subsection{Circular por ahí}

Los curiosos suelen anotarse en sus primeras clases de actuación siguiendo consejos de conocidos - "un amigo que estudiaba teatro"-, en general yendo a estudiar al mismo lugar que él o ella; o por algún criterio de cercanía o de los lugares que frecuentan - para aquellos que comienzan a estudiar durante la secundaria, un taller que se da en la escuela, o en otros casos, un centro cultural del barrio o al que la persona frecuentaba desde antes, por alguna razón-. Además, el Centro Cultural San Martín y el Centro Cultural Rojas, que ofrecen, entre tantos otros, talleres de actuación, parecen ser lugares con mucha visibilidad para las personas que quieren empezar a hacer teatro, ya que varios mencionan haber tomado sus primeras clases allí.

Deciden empezar a estudiar actuación por motivos muy variados que pueden ir desde "ver qué onda"o "explorar su lado artístico"-en ocasiones por recomendación del terapeuta-, hasta porque están convencidos de que la actuación es su vocación y/o porque tienen la fantasía de "ser famosos" y actuar en la tele o en el cine. En los casos en que deciden que se van a dedicar a la actuación a tiempo completo, suelen despertar oposición por parte de su familia, que en algunos casos los desalienta por ser una "profesión sin futuro ni salida laboral", y que en cambio les sugiere una carrera universitaria "más tradicional".

Para los recién llegados, el universo del teatro alternativo es la clase en la que estudian, su maestro y sus compañeros. Escuchan que algunos de ellos hacen referencia a una serie de nombres (Alezzo, Angelelli, Bartís, Catalán, Chávez, Daulte, Fernandes, Kartun, Savignone, Tolcachir, Veronese, entre otros) que no les dicen mucho. No pueden diferenciar dramaturgos, actores o directores. Tampoco saben quiénes dan clase y quiénes no, ni pueden tan 
claramente a aquellos que tienen más trayectoria. A lo sumo, si vieron alguna obra en la que actuaba o dirigía uno de esos u otros nombres, los relacionan. No tienen mucho más criterio que lo que le gusta - de aquello que conoceny lo que alguien que parece saber más les recomendó. Si siguen yendo a clase es porque la pasan bien y se divierten, porque el maestro o la maestra y sus compañeros les caen bien y/o porque hay un chico o una chica que les gusta. Pero también es muy común que se cambien a otro taller u otra escuela -a partir de recomendaciones que reciben de gente que van conociendo-, en algunos casos cada seis meses, o cada un año, o incluso que estudien en más de un lugar a la vez. También, con los amigos que hacen en la clase, van a ver obras, recorren los teatros del teatro alternativo, se fascinan y se pierden en un universo del que no habían imaginado sus dimensiones; quizás, en ese recorrido alguno de ellos descubre un actor o director que le gusta, descubre que da clases y decide estudiar con él.

En no pocas ocasiones, esta circulación implica un cambio de ciudad. En muchos casos, la migración suele darse al terminar la secundaria, con ayuda familiar y con algún otro motivo que la justifique en términos morales -generalmente, estudiar una carrera universitaria más tradicional-. Cuando esa migración se da exclusivamente para estudiar teatro, los estudiantes suelen anotarse en la carrera de actuación de las universidades públicas de Buenos Aires, la Escuela Metropolitana de Arte Dramático (EMAD) o en la Universidad Nacional de Artes (UNA). En ambas instituciones hay un examen de admisión que es muy competitivo y que deja afuera a muchos aspirantes que en esos casos deciden anotarse en una institución privada. También, algunos de los estudiantes que entran en estas instituciones estudian, una vez por semana, en un estudio privado.

\subsection{Ser cautivado por un maestro}

En un determinado momento de esa circulación, algunos estudiantes se encuentran con un maestro que los cautiva -en un doble sentido: los deslumbra y los retiene - con su impronta: el entrenamiento que propone, la mirada del maestro y su forma de encarar el teatro, todo los fascina. En algunos casos, estos estudiantes llegan a ese taller desprevenidos, apenas por alguna recomendación o algún dato que despertó su curiosidad; en otros, quedaron deslumbrados 


\section{Aprendiendo a lo largo de la ciudad...}

por una obra que vieron y decidieron entrenar con el director o con alguno de los actores. Retrospectivamente, los actores suelen mencionar este momento como el momento en que empezaron a hacer teatro en serio.

Recién llegados a este nuevo estudio, los estudiantes no comprenden bien: tratan pero no consiguen actuar en un estilo que están lejos de dominar y entienden apenas algunas de las cosas que el maestro dice. Pero están fascinados, dejan de peregrinar de un taller a otro y se quedan y estudian con ese maestro por un período largo, en general de dos a cuatro años, como mínimo. Los estudiantes que han sido cautivados quieren entender todo lo que el maestro dice, hacer bien a sus ojos, y es casi inevitable que empiecen a ver y a hablar como él, también porque la actuación supone nombrar una serie de cuestiones de las que no se habla en la vida cotidiana y para las que hace falta un lenguaje específico.

De a poco, en un proceso que puede durar años, los estudiantes empatizan y empiezan a sintonizar una sensibilidad similar a la de su maestro, y que se manifiesta en una forma de mirar, pensar, hablar gozar, sentir, hacer y querer hacer teatro. El proceso de aprendizaje es, sin embargo, lento. Aprender las sutilezas del estilo de actuación que el maestro enseña, jerarquizar lo importante de sus palabras, pero también, los matices de sus opiniones en el tono de su voz, y lo que quiere decir con lo que dice, lleva tiempo. El aprendizaje también es lento porque la clase de teatro, a diferencia de otro tipo de clases, no es un lugar donde las personas estén constantemente preguntando lo que no entienden.

También es común que los estudiantes empiecen a pasar más tiempo con su maestro en este período, ya sea tomando más de una clase con él o comiendo con él y otros compañeros después de las clases. Escuchan atentamente cada palabra del maestro, le piden recomendación y consultan su opinión sobre obras, actores y directores. También van a verlo actuar o dirigir. A medida que ganan experiencia, y luego de unos años de entrenamiento, algunos de los estudiantes pasarán a ser asistentes de dirección en obras de su maestro, a asistirlo en sus clases, a dar clases en su estudio y/o a actuar en alguna de sus obras. No es que algunas de estas cosas no sucedieran con los maestros anteriores; es que ahora todo sucede al mismo tiempo y con mayor intensidad.

Algo así le pasó a Marcelo. Marcelo nació en la Ciudad de Buenos Aires en 1989 
y de acuerdo a los maestros que menciona, se formó principalmente en el circuito del teatro de estados (ver esquema 2 en página 14). Después de estudiar teatro en varios lugares en los que no se terminaba de enganchar, quedó fascinado con las clases de clown de Pablo de Nito en el Centro Cultural San Martín, con quien después siguió estudiando teatro, en un espacio que quedaba por Chacarita. Esas clases eran más largas y sentía que profundizaba más. En el 2008, Pablo de Nito puso su propio estudio, La Gaya Teatro, en Congreso, a cinco cuadras de la casa de Marcelo. "Lo cual es como que te pongan el kiosco [al lado de tu casa]" me dice Marcelo, "yo fui un montón y ya era parte de los grupos. Después sucedió un montón, hacer clown, hacer teatro, asistir sus clases, asistir sus obras, actuar en las varietés y las muestras que organizaba el estudio"(Marcelo, comunicación personal, 2016). Asistió una obra en la que actuaba De Nito -El oso-, y otra que este maestro dirigía - El chancho- en la que participó de todo el proceso de ensayo -que duró tres años- aunque después se tuvo que ir porque estaba empezando a ensayar una obra en la que actuaba y se le superponían los horarios. Hablándome de Pablo, Marcelo me decía: "Si estudiás mucho con alguien, y compartís mucho, empezás a ver eso [el teatro] desde el mismo lugar" (Marcelo, comunicación personal, 2016).

Para este momento, la fantasía de los estudiantes se ha reorientado, al menos parcialmente: si, originalmente algunos estudiantes habían comenzado a actuar porque querían "ser famosos" - estar en el cine o en la televisión-, ahora una de sus ansias mayores es ser llamados por su maestro para actuar en una obradirigida por él. Del mismo modo, el universo del teatro alternativo comienza a tomar un lugar más importante en las expectativas de los estudiantes, que en general buscan participar en obras y actuar -aunque esto también puede haber sucedido en el momento anterior-.

Algunos de los compañeros con los que los estudiantes se encuentran en la clase también tienen un rol central en este proceso. Encontrarse con compañeros que también están enganchados con la propuesta del maestro, que quieren trabajar en serio, es decir, sobre todo, juntarse a ensayar y a preparar trabajos para mostrar en clase y compartir, entre otras cosas, la excitación de exponerse a la mirada del maestro, es una parte importante del proceso. En muchos casos, de esas amistades surgen grupos de trabajo que luego preparan 


\section{Aprendiendo a lo largo de la ciudad...}

y llevan a cabo obras de teatro en un estilo de actuación similar al que su maestro propone -incluso cuando el maestro no coordina ni dirige esos trabajos-. Ludmila, que nació en 1981 en Baradero y por los maestros que menciona se formó principalmente en el circuito del teatro de estados cuenta que, en sus primeros años, fue muy importante para ella encontrarse con Marcos y Julián, otros dos estudiantes de actuación -uno más grande y el otro más chico que ella-, con los que realizó varias obras. Comenzó preparando una escena en una clase, después uno de ellos la llamó para asistir una obra que estaba haciendo y para actuar. Ludmila afirma que son dos personas de las que aprendió y sigue "tratando de aprender todo el tiempo, desde el trabajo, eso, mucho trabajo, de que hay que trabajar y hay que trabajar y hay que trabajar y hay que trabajar" (Ludmila, comunicación personal, 2016).

El momento de ser cautivado por un maestro no es una experiencia ligada de manera unívoca al goce: los estudiantes también la pasan mal por la frustración que supone darse cuenta que no están consiguiendo actuar como el maestro pide. Mariela —nació en 1975, en Capital Federal y por los maestros que menciona se formó principalmente en el circuito realista (ver esquema 1 en página 13) — cuenta que cuando empezó a estudiar en las clases de Chávez era muy joven, acababa de salir de la escuela secundaria: "yo era..., tengo una fiesta, no voy a teatro; tengo tal cosa, no voy a la clase". Venía de ser, en la escuela, "la loca", la graciosa, la que imitaba a los profesores bien y hacía reír a todos, de ahí creyó que tenía que ser actriz. "Julio me bajó de un hondazo", dice, "él era muy duro conmigo". Sin embargo, se sentía muy identificada en "cómo él veía al humano, su manera de mirar el almita humano, con una pasión por lo humano, pocas veces visto".

Con el pasar de los meses, se dio cuenta que todo lo que ella creía sobre el teatro, no tenía nada que ver con lo que tenía que empezar a hacer. Entendió que la actuación era otra cosa: el compromiso, el trabajo, Julio le enseñó el valor del trabajo, que tenía que "entrenar, entrenar, entrenar. No entrenaba para el aplauso, ni para la gloria, ni nada. A partir de ese momento nunca paré, no existió otra cosa”. Algún tiempo después, "yo ya estaba tomada, y si me tenía que quedar hasta las tres de la mañana haciendo la escenografía, 
me quedaba.

"Mariela recuerda la adrenalina de esos años, el momento de pasar y tener que actuar frente a su maestro, la necesidad de aprobación, cómo le latía el corazón, fuerte. "Sentía que me iba a morir", exagera. O la emoción que le daba ver actuar a su maestro -hasta hoy le pasa-, y las ganas de actuar como él que le entran cada vez que lo ve.

Algunos años después, Mariela fue a estudiar con Augusto Fernandes, a quien Chávez siempre mencionaba como su principal maestro -“Augusto, Augusto, siempre hablaba de Augusto; yo sabía que en algún momento, cuando terminara con él iba a ir con Fernandes"-. En esa misma época, Chávez también volvió a entrenar con Fernandes. Mariela y Chávez fueron compañeros. "Un día viene Julio [Chávez] y me dice -y lo imita con una voz entre vergonzosa y casual, como si le preguntara algo sin importancia, si quería un café_- ¿Querés hacer una escena conmigo?” Esa noche no dormí. Hicimos Otelo y Desdémona” (Mariela, comunicación personal, 2017).

El momento de quedar cautivado por el maestro es, como en el caso Mariela, un período de cambio profundo de valores, de la comprensión de lo que es el teatro y su relación con términos como el trabajo, el compromiso y la "fe". Los estudiantes suelen referirse a la "fe" que adquieren en el trabajo, designando la creencia de que eso que están haciendo -actuartiene un profundo valor, y que vale la pena dedicarle decenas y decenas de horas. Esta "fe" viene de la mano de una ética del trabajo que el maestro consigue inculcar en algunos de sus alumnos, una disciplina, y una entereza de persistir y continuar insistiendo ante la frustración. Es a esa persistencia a la que Ludmila y Mariela se refieren cuando insisten en que hay que trabajar y que hay que entrenar. Los estudiantes suelen apreciar la rigurosidad del maestro y manifestar una fuerza que los impulsa a seguir trabajando y esforzándose a pesar de las frustraciones y las palabras duras5. Así lo manifiesta Franco, un estudiante del circuito realista, que mientras estudiaba con Alezzo también estudiaba en otro lugar, en el que no encontraba la misma rigurosidad. 


\section{Aprendiendo a lo largo de la ciudad...}

La sensación era que en lo de Alezzo me comía un lomo con fritas y un buen helado, y en [el otro lugar] me comía un yogur descremado. (...) Lo que yo veía en lo de Alezzo era que [- durante las devoluciones, después de actuar-] a unos los mataban, a otros les decían estupendo y a otros bien pero... ,y les marcaban cosas y no se dejaba pasar nunca una. Entonces yo pasaba [a actuar] tranquilo, porque si me dicen que está bien es porque es el camino de lo que quieren que haga. (...) Y no, como pasaba en [el otro lugar], esta cosa de "empecemos por lo que está bien". Que yo entiendo, si es una cosa vocacional, de barrio, y la gente va a divertirse y nada más, bueno, decile que está todo bien, marcale alguna que otra cosa y así te paga la cuota el mes que viene, y se va contento. Pero en un lugar de formación del actor, me parece que tiene que haber otra rigurosidad, entonces yo veía una rigurosidad en lo de Alezzo que era implacable, que cuando te tenía que defenestrar te defenestraba, y cuando te tenía que alabar te alababa y cuando te tenía que marcar alguna cosa te la marcaba. (Franco, comunicación personal, 2016).

Cautivados por su maestro, los estudiantes siguen circulando por el teatro alternativo, miran todo tipo de propuestas teatrales, se presentan en castings y/o ensayan una obra. En todo lo que hacen, su mirada y su forma de querer hacer teatro están cada vez más teñidas por la visión de su maestro. Sin embargo, eso no quiere decir que los estudiantes adopten, necesariamente, una actitud purista. Otra necesidad empieza a invadirlos: la de querer actuar. Y quizás encuentran una obra en la que el director tiene una concepción totalmente diferente de la actuación a la de su maestro, como le pasó a Mariela.

Fue en esas épocas que estaba desesperada por actuar, por subirme a un escenario como sea, como sea. Y aparece una amiga, de lo de Julio [Chávez], y me dice, hay una obra que se les cayó una actriz.(Mariela, comunicación personal, 2017).

Mariela cuenta que leyó la obra y no entendió nada. 
Viste esas obras, que en esa época más todavía, en una época que cuanto más rara mejor, si no entendías un carajo mejor, si el público no entendía, mejor. ¿Dónde están los personajes? ¿Qué dice mi personaje? Dice unos diálogos pesadísimos, complejísimos - y en el modo en que alarga en estas últimas dos palabras la pronunciación de las í acentuadas, en realidad en el tono de todo lo que dice, Mariela da a entender que le parecía muy mal, que no era para nada el tipo de teatro que a ella le interesaba o le parecía bueno-. Cuando llegué a hablar [con el director] le digo: me encantó la obra. Mentira, mentira. Mentí todo. No entendí nada. (Mariela, comunicación personal, 2017).

Cuando iban a hacer la obra, antes de actuar, el director les hacía hacer unos ejercicios

y yo no creía nada en esos ejercicios. Pero los hacía -y me aclara en un susurro - porque quería actuar. [El director] decía, paso la energía, y yo decía, esto no lo hicimos nunca con Julio [Chávez], no paso ninguna energía. No creo nada en esas cosas, pero sí me parece muy importante la fe, entonces digo, el que tiene fe, adelante. Entonces era $-\mathrm{y}$ hace algunos gestos moviendo las palmas de sus manos delante de su torso, como mostrándome cómo pasaban la energía- y yo hacía, era muy buena haciendo lo que el otro me proponía, porque yo sentía que nadie se daba cuenta que no era mi palo, pero para mí eran raros. (Mariela, comunicación personal, 2017).

En esta instancia Mariela estaba tomada al mismo tiempo por la necesidad de actuar y por la visión del teatro de su maestro. La formación en un estilo de actuación stanislavskiano como el que Mariela había aprendido con Chávez, pone mucha importancia al análisis y la comprensión de los personajes al momento de leer una obra, cosa que Mariela no podía hacer en la obra en la que la habían invitado a participar, porque era una obra "rara". Todo lo que ellos hacían - ¡hasta los ejercicios de calentamiento antes de actuar! - lo veía con desconfianza, como un tipo de teatro diferente en el que ella creía. Con todo, sus ganas de actuar eran fuertes, y pudo pretender 


\section{Aprendiendo a lo largo de la ciudad...}

estar encantada con la obra y con el grupo.

Esquema 1: Los maestros activos en Buenos Aires del linaje realista hacia

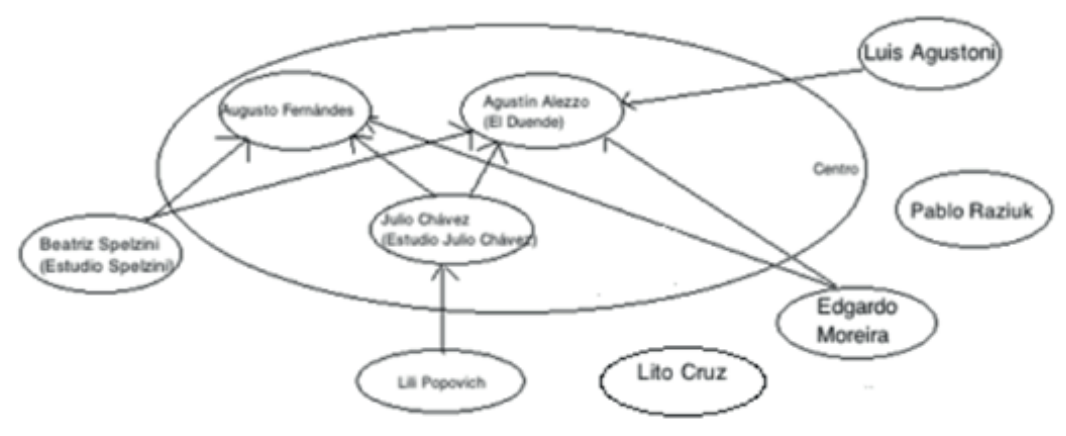

Fuente: Elaboración propia

Las flechas señalan con quien se formó cada maestro (en caso de que el maestro del maestro se encuentre en el esquema). Aquellos que se encuentran al interior del círculo central son los maestros más renombrados de este linaje, es decir, aquellos por los que, en la época, se estudiaba con mayor frecuencia.

Como veremos en el próximo apartado, al cautivar a algunos estudiantes el maestro los inicia en un circuito en el que aquellos seguirán circulando por algunos años. Como también veremos en seguida, esa circulación posterior se suele dar entre los maestros más renombrados de cada linaje -los que se encuentran al interior de los círculos centrales en los esquemas 1 y 2 En cambio, el momento que aquí estoy describiendo, el de quedar cautivado por un maestro que inicia al estudiante en una visión de la actuación que implicará, entre otras cosas, su posterior circulación al interior del circuito, suele darse por un maestro que, si bien pertenece al linaje, no es uno de los maestros centrales. A continuación, me refiero a estos otros maestros - ex alumnos de uno o varios de los maestros centrales, o de maestros fallecidos o fuera de la actividad- como maestros periféricos (en los esquemas 1 y 2 los maestros que están fuera del círculo central son algunos de esos maestros periféricos, los que aparecieron mencionados con mayor frecuencia por mis entrevistados). 
El hecho de que estos maestros periféricos -y no los centrales - tiendan a ser los que cumplen el rol de iniciar a un estudiante en una comprensión de la actuación que implicará su posterior circulación en un circuito, se debe a dos motivos. Por un lado, a un cierto régimen de visibilidad e invisibilidad de las diferentes escuelas, que es diferente en distintos momentos de la carrera secuencial que aquí presentamos. Para quien no hace mucho que estudia teatro, es menos probable conocer a los maestros centrales de cada linaje, ya que estos no hacen ningún tipo de publicidad. Su única publicidad son sus estudiantes -y sus ex alumnos, algunos devenidos maestros periféricos de su circuito-. En cambio, los maestros periféricos suelen no tener su propioestudio, o, si lo tienen, también dan clases en centros culturales o clubes barriales que tienen mayor visibilidad, como hemos visto en la sección anterior, para quienes recién están dando sus primeros pasos en la actuación.

Esquema 2. Los maestros activos en Buenos Aires del linaje del teatro de estados hacia 2016-2017.

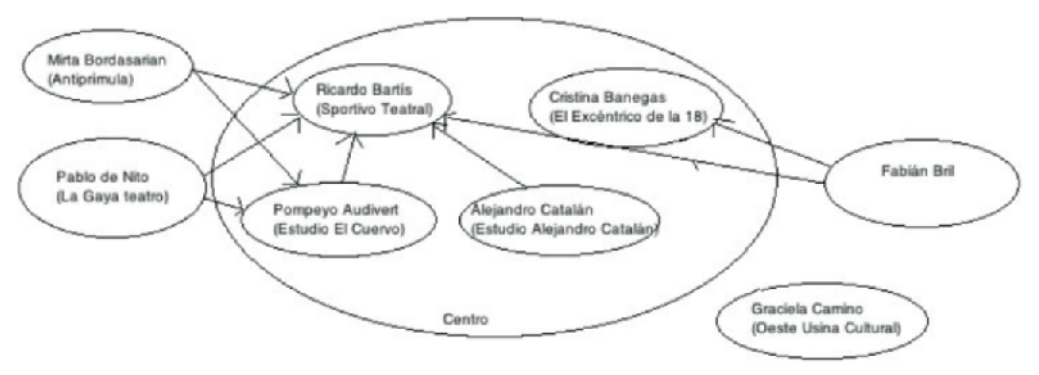

Fuente: Elaboración propia

Las flechas señalan con quien se formó cada maestro (en caso de que el maestro del maestro se encuentre en el esquema). Aquellos que se encuentran al interior del círculo central son los maestros más renombrados de este linaje, es decir, aquellos por los que, en la época, se estudiaba con mayor frecuencia.

$\mathrm{Si}$, con todo, un estudiante que no hace mucho que estudia teatro llega a la escuela de alguno de los maestros centrales de uno de los circuitos, lo más probable es que tenga que empezar por tomar clases en grupos para principiantes -o para recién llegados al estudio-, que no suelen estar dadas por el maestro central. Pasarán uno o dos años hasta que el principiante pueda estudiar con el maestro principal del estudio, el maestro central del circuito. Antes de eso, el estudiante se encontrará con un maestro periférico del circuito que, en muchos casos, ocupará el lugar de ese maestro que primero 
cautiva al estudiante.

Por cualquiera de estos motivos el maestro que primero inicia a un estudiante en un circuito no es necesariamente -de hecho, como venimos explicando, no lo es la mayoría de la veces - aquella persona a la que el estudiante retrospectivamente, luego de formarse por varios años- más respeta por su trayectoria, ya sea como actor, como director e incluso como pedagogo. Sin embargo, buena parte de los estudiantes tienden a recordar con una intensidad particular el fervor que sintieron por ese primer maestro que los introdujo en una forma de entender el teatro y suelen afirmar que la intensidad con la que su forma de entender el teatro se mimetizó con la de ese maestro, fue mucho mayor que lo que le sucedió con los maestros con los que estudiaron después, aún cuando a estos últimos les reconozcan una mayor trayectoria.

Por último, es preciso aclarar que si bien las historias que aquí he descrito están principalmente centradas en las clases de actuación, el momento de quedar fascinado con un maestro también puede ocurrir durante el ensayo de una obra.

\subsection{Circular al interior de un circuito}

Luego de la profunda influencia que el maestro deja sobre los estudiantes cautivados por su mirada durante el segundo momento, ya nada es igual. Los estudiantes continúan circulando por otros estudios, pero el criterio en la selección de estos ya no está ligado a una cercanía ni a otras instituciones que frecuentan o a cualquier tipo de recomendación que reciben. Convencidos de una cierta comprensión del teatro, los estudiantes quieren seguir formándose en el mismo estilo de actuación.

Durante su formación los estudiantes van reconstruyendo algunos elementos de la historia de la actuación porteña, teniendo como eje el linaje al que pertenece su maestro. Así es que comienzan a descubrir quién o quiénes fueron los maestros de su maestro, así como a otros actores y directores formados en el mismo linaje. En su trabajo sobre el aprendizaje del canto en estilos que el autor engloba bajo el término música hindustani, Rahaim (2008) ha prestado atención al modo en que, durante el aprendizaje, el estudiante va desarrollando 
un cuerpo parampara (término que en sánscrito significa linaje), lo define como "una forma de ser musicalmente hablando, formada a partir de disposiciones que son transmitidas tácitamente a través de un linaje de enseñanza" (p. 340, la traducción es mía). En el caso de los estudiantes de teatro ocurre que, luego de haber estudiado con un maestro que los ha cautivado profundamente $-\mathrm{y}$ con el que han aprendido una sensibilidad que es la del linaje en el que su maestro está inscrito-, los estudiantes continúan circulando entre maestros que no solo enseñan el mismo estilo de actuación, sino que también pertenecen al mismo linaje.

Lo más común es que los estudiantes continúen su camino yendo a estudiar con alguno de los maestros de su maestro, lo que también suele implicar un desplazamiento de la periferia al centro del circuito. En algunos casos, esto se da naturalmente al interior de una escuela, cuando un estudiante pasa de estudiar con alguno de los maestros que dan las clases para principiantes -y recién llegados - a estudiar con el maestro principal de ese estudio. En otros casos, ese desplazamiento es parte de una migración hacia la Ciudad de Buenos Aires, donde enseña el maestro del maestro. Esto se debe a que algunos maestros periféricos de cada circuito enseñan en ciudades de la Provincia de Buenos Aires o en el interior del país. A diferencia de los estudiantes que migran en el primer momento de la carrera secuencial, quienes llegan a la Ciudad de Buenos Aires luego de haber sido cautivados por un maestro ya cuentan con algunas referencias sobre el teatro porteño, y sobre el o los maestros con los que quieren estudiar. En algunos casos, también, los estudiantes no se mudan, sino que viajan semanalmente a Buenos Aires para estudiar con el maestro de su maestro, algo que sucede desde ciudades como La Plata que se encuentra a 60 kilómetros de la Capital Federal — ver del Mármol (2016) - y Rosario, que se encuentra a 300 kilómetros6.

El desplazamiento hacia el centro del circuito supone encontrarse con una mayor cantidad de estudiantes -y en general, más experimentados-que también se encuentran en esta instancia de su formación y por lo tanto, comparten una visión similar del teatro y la actuación. En esa circulación, los estudiantes siguen profundizando en el estilo de actuación que promueven los maestros del linaje al que ahora adhieren. 
En cambio, volver de los maestros del centro hacia los maestros de la periferia es algo poco frecuente en esta instancia, a no ser cuando se vuelve a entrenar con el maestro que cautivó al estudiante en el segundo momento. $\mathrm{O}$, cuando se debe a razones económicas, ya que los maestros centrales del circuito suelen cobrar una cuota más cara.

La carrera secuencial que aquí presento no incluye directamente a los estudiantes de las instituciones públicas de artes dramáticas — la Universidad Nacional de las Artes (UNA) y de la Escuela Metropolitana de Arte Dramático (EMAD) - , ya que estas instituciones ofrecen un tipo de formación que tiende a cierto eclecticismo, proponiendo al estudiante maestros que enseñan distintos estilos de actuación en cada año. Por lo tanto, las trayectorias de estos estudiantes suelen ser bastante diferentes. Sin embargo, es preciso hacer algunas salvedades. En primer lugar, muchos de los estudiantes que asisten a estas instituciones también estudian, una vez a la semana, con un maestro de una institución privada. Asimismo, en algunos casos, uno de los maestros de estas escuelas cumple el rol de iniciar al estudiante en uno de los circuitos. Así, los estudiantes pueden seguir asistiendo, paralelamente a su formación en la institución pública, con ese maestro en su estudio privado o con otros maestros del circuito en el que aquel los inició. Por último, para el caso de la UNA, donde los estudiantes pueden elegir entre diferentes cátedras de actuación, los estudiantes que ya se encuentran en esta instancia - la de circular al interior de un linaje al que adhieren-, tienden a elegir a los maestros que consideran más cercanos a su estilo de actuación.

En esta instancia, todo el mar de nombres - de actores, directores y dramaturgos - que circulan en las conversaciones con otros estudiantes comienzan a organizarse, a cobrar sentido. No cualquier sentido. El linaje al que los estudiantes ahora adhieren es su principal punto de referencia, y los maestros más vinculados a este son los que los estudiantes conocen más en detalle. Los nombres de los maestros que en un linaje merecen el mayor respeto pueden ser vistos como parte del pasado en otro, referidos con ironía o resultar indiferentes o desconocidos.

También, los estudiantes han ido viendo y participando de obras, y conociendo actores, directores y dramaturgos. Van mucho a ver actuar a otros 
compañeros y amigos que también son actores. Muchos de esos compañeros tienen trayectorias de las más variadas (no olvidemos que no todos los estudiantes que se encuentran en un estudio han seguido la carrera secuencial que describimos aquí, ni circulan por el mismo circuito). Mucho de lo que ven, de lo que sienten y piensan, del modo que disfrutan - o no- cuando ven obras, se encuentra muy en la clave del modo de entender la actuación en el que los estudiantes se están formando. En esta etapa todavía temprana, su visión tiende a ser "ortodoxa", "fundamentalista"; sin embargo, como veremos en la sección siguiente, pueden ocurrir sorpresas, cambios de dirección inesperados.

En varietés, después de obras o por conocidos de conocidos, los estudiantes conocen a otros actores con otras trayectorias. Los ven actuar y relacionan esas actuaciones con ciertos maestros porque, como suelen decir los estudiantes, en la forma de actuación se puede ver, a veces, con quién estudió o estudia un actor. No es raro que, a poco de conocerse, dos estudiantes conversen sobre su formación.

Leandro nació en 1970 en Buenos Aires y por los maestros que menciona se formó principalmente en el circuito realista. Tiene cuarenta y seis años aunque parece menos. Lo conocí en el cumpleaños de Laura, una amiga que tenemos en común, que había contratado a otras dos personas para que hicieran un show teatral. El show incluía la participación del público, y nuestra amiga nos había avisado que probablemente nos eligieran a nosotros, porque habíamos estudiado teatro. Después del show, en el que participamos, conversamos un rato. Me dijo que él había estudiado mucho con Lili Popovich y que le interesaba principalmente el realismo. Cuando dijo Lili Popovich lo miré con cara de que ese nombre no me sonaba para nada y aclaró: "Ella es del estudio de Julio Chávez, se formó con él. Después yo también estudié con él”. (Leandro, Comunicación personal, 2016). Leandro empezó a estudiar teatro a los treinta y tres años con Lili Popovich, a principios de los dos mil. Después estudió con Chávez y con Alezzo - maestros centrales del circuito-, pero, luego de un tiempo, regresó a estudiar con Lili, a quien considera su principal maestra.

Neuman (2004, citado en Rahaim, 2012) ha señalado que, en la tradición de música hindustaní, es muy común que cuando un estudiante se presenta o es presentado, de cuenta de su identidad mencionando al maestro con el que estudia. 
Entre los estudiantes de teatro, esta situación es similar con elagregado de que, cuando se trata de un maestro periférico de un circuito -como lo es Lili Popovich del circuito realista (ver esquema 1)-, se suele mencionar al maestro de ese maestro, que en general es alguien conocido. Así, los linajes funcionan, también, como una forma de dar sentido y organizar a algunos de los nombres que circulan en el teatro alternativo.

Si bien la circulación durante este tercer momento viene dada en buena medida por la búsqueda consciente del estudiante de mantenerse al interior de un linaje de actuación, también es cierto que las trayectorias pasadas condicionan, al menos en alguna medida, los maestros a los que se puede acceder.

Los maestros centrales del circuito suelen recibir muchos estudiantes y en algunos casos toman algún tipo de prueba para seleccionar a los estudiantes de sus clases. Leandro cuenta que haber estudiado con Chávez le abrió muchas puertas. Así, cuando fue a estudiar con Alezzo, la simple mención de haber estudiado con Chávez le valió que lo aceptaran en el seminario para actores de este maestro, sin tener que realizar una prueba. Durante mi trabajo de campo, asistí a una reunión informativa que Alezzo daba para las personas que querían entrar a hacer el seminario de actuación con él, es decir, actores con experiencia que querían estudiar directamente con él, sin hacer la formación de cuatro años en su escuela. Luego de hablar un rato sobre la dinámica de la escuela, del taller y de la prueba que deberían realizar los aspirantes -que suponía mostrar una escena de unos diez minutos con otro actor o actriz-, Alezzo conversó algunos minutos con cada aspirante. Cada uno a su turno, pasaron a sentarse en una mesa frente a él y le contaron un poco sobre su formación. Entre ellos pasó una chica que, si bien había estudiado teatro durante varios años, dijo nunca haber trabajado con texto. Alezzo le dijo que en ese caso no iba a poder estudiar con él, y que no tenía sentido que hiciera la prueba. Ella protestó pero acabó por aceptar.

Al rato pasó otro actor. Mientras él le contaba sobre su formación, Alezzo lo miraba. "Yo a vos te conozco de algún lado", le dijo. Después de un breve intercambio coincidieron en que Alezzo lo había visto actuar en una obra dirigida por Chávez que estaba en cartelera en ese momento. Alezzo le dijo que su trabajo le había parecido muy bueno, 
que no hacía falta que pasara la prueba, que él ya estaba aceptado para formar parte del seminario (Libreta de campo, marzo 2015).

Como vemos, la posibilidad de estudiar con ciertos maestros, no depende únicamente de la voluntad de los estudiantes de entrenar con uno o con otro. La trayectoria pasada de los actores no condiciona solo con quién quieren estudiar sino también, en alguna medida, las credenciales que permiten estudiar con algunos maestros -sobre todo los maestros centrales de los circuitos-. Si bien no asistí a la prueba que se hizo, es evidente que aquellos actores entrenados en un estilo de actuación stanislavskiano -incluso aunque no vinieran circulando en el circuito realista-, tuvieron más facilidad para realizar la prueba y ser aceptados en su seminario.

Claro que los estudiantes siempre tienen la opción de entrar a la escuela a estudiar con otro maestro, y solo con el tiempo -quizás luego de uno o dos años - pasar a estudiar con el maestro principal de la escuela. Pero a veces los estudiantes, por su formación pasada, consideran que ya tienen una cierta experiencia y no tienen ganas de pasar uno o dos años estudiando con un maestro menos reconocido antes de poder estudiar con el maestro al que venían a buscar.

\subsection{Distanciarse del maestro: la propia visión}

Desde el momento en que un estudiante es cautivado por un maestro hasta el final de su posterior circulación por el circuito en que este se halla inscrito pueden pasar en torno a unos siete años. La mayoría de las veces, solo después de un largo tiempo de aprendizaje y de adherir a una cierta visión de la actuación los estudiantes comienzan a distanciarse de la mirada de sus maestros -en grados y formas variables-, y desarrollar una impronta, una visión o una síntesis más personal. No se trata simplemente de que el estudiante hace lo que quiere; la reformulación o síntesis personal a la que me refiero, la de aquellos que han sido cautivados por períodos largos y de manera intensa por una forma de concebir la actuación, es sutil: la influencia de su maestro es profunda y persiste, pero es objeto de una síntesis más o menos singular. 
Este momento es el más difícil de observar etnográficamente y ocurre mientras los estudiantes siguen circulando por otros roles en el teatro - en particular si devienen directores o maestros - , o por otras disciplinas, o si siguen entrenando pero en otros estilos de actuación. A continuación, haré mención de tres de estos casos pero, por falta de espacio, solo desarrollaré uno de ellos.

En primer lugar, esta síntesis puede ocurrir cuando un estudiante deviene maestro $y$, si bien empieza a enseñar de un modo similar al de sus maestros, también utiliza las clases como forma de experimentación y comienza a introducir sus propias variantes. Estas variantes no son conceptos o ideas abstractas, sino técnicas muy concretas a través de las cuales el -ahora-maestro va buscando guiar a sus estudiantes hacia un cierto estilo de actuación que, aunque se inscribe en una tradición también es, en alguna medida, personal. El caso de Alejandro Catalán, tal como lo desarrollé en otra parte (Battezzati, 2017) es una síntesis de esta clase para el caso del circuito del teatro de estados.

También, en algunos casos, los estudiantes siguen circulando por otras disciplinas, no sólo la actuación. Las enseñanzas de su maestro sonreinterpretadas en estos contextos. Cuando esto sucede, el estudiante es consciente de que el modo en que rearticula las enseñanzas de su maestro es contradictorio con el modo en que su maestro las entiende; sin embargo, asegura que, en el fondo, es lo mismo, visto desde otra perspectiva. Es decir, el estudiante sostiene que, aún cuando lo que hace presenta una contradicción con lo que su maestro cree, hay una relación de continuidad con las formas más sutiles de entender o interpretar sus enseñanzas en otro contexto. En otra parte (Battezzati, 2017) he descrito cómo estudiantes de actuación rearticulan y/o redescubren las enseñanzas de sus maestros al practicar otras disciplinas.

Finalmente, existe un tercer tipo de síntesis personal. Este se da cuando, después de circular durante mucho tiempo - siete, ocho años- por un circuito, el estudiante decide seguir entrenando con un maestro que enseñe un estilo de actuación diferente. Por lo que he podido ver, esto es más común entre los estudiantes del circuito realista.

Agustina nació en 1988 en La Plata. Empezó a estudiar teatro en la primaria, en un instituto de arte al que la familia la mandaba a contra turno. 
En la secundaria siguió con maestros de ese instituto. Estudió dos años con Gastón Marioni. Cuando terminó la escuela se anotó en el IUNA, pero no pasó el examen de admisión. Le recomendaron un maestro para estudiar, "Dalezio". Lo buscó en Google y no encontró nada. Agustina quería estudiar con alguien reconocido. Cuando volvió a hablar con la persona que se lo había recomendado le aclaró: "es Alezzo, no Dalezio". Igual Agustina no lo conocía: cuando venía a Buenos Aires a ver teatro, iba a ver teatro comercial, el de Avenida Corrientes; desconocía el teatro alternativo.

Se anotó, hizo toda la formación, cuatro años en total, los primeros dos con Lisardo Laphitz, los siguientes con Alezzo. Hizo tres años más de seminario para actores con Alezzo. Los primeros tres años iba y venía a Buenos Aires; después se mudó. No volvió a probar de entrar al IUNA. Pero, como quería tener una formación teórica complementaria, se anotó en Artes, en la UBA.

Entonces cuando yo leía en la facultad, que igual lo sigo sosteniendo hasta hoy -se ríe, una risa corta-, pero puedo abrir un poco más algún que otro cuestionamiento, que hay métodos que para algunos trabajos del actor no sirven tanto. Pero en su momento leía que el método stanislavksiano formaba a los actores en una cosa psicologicista que hacía que el actor se metiera para adentro y yo decía, no entienden nada, así que me peleaba con los textos mientras los estaba estudiando para la facultad-, y mientras dice eso hace gesto de subrayar con bronca los textos en el aire (Agustina, comunicación personal, 2017).

En ese entonces, Agustina estaba convencida que el tipo de entrenamiento que hacía era el mejor. También empezó a hacer castings para publicidad. Ya venía trabajando en varias obras, primero en algunas de teatro infantil, después en otras de teatro alternativo. Cuando hacía tres años que estaba haciendo el seminario con Alezzo, él la llamó para hacer Los justos de Albert Camus. También, en uno de los seminarios que hizo con Alezzo, fue elegida para hacer un monólogo del personaje de La Luna en Bodas de sangre, de Federico García Lorca.

Entra La Luna en escena y empieza a decir un montón de cosas. $\mathrm{Y}$ claro, es una obra que es simbolista, entonces los personajes 


\section{Aprendiendo a lo largo de la ciudad...}

como La Luna, no son realistas. Él [Alezzo] me decía, "usted lo dice muy bien, se entiende todo lo que dice, pero es otra cosa esto, es de otra manera", y yo no podía llegar, porque tenía que usar otra voz, otra forma de poner el cuerpo, una cosa que no me terminaba saliendo. (Agustina, comunicación personal, 2017).

Retrospectivamente, Agustina cuenta este hecho como parte de una curiosidad o una necesidad por complementar su formación con otro tipo de entrenamiento. Agustina había visto dos obras de Marcelo Savignone que le habían gustado: Un Vania y Suerte.

Lo que me llamó la atención era el trabajo físico que él hacía, él y con los otros actores, que era un trabajo muy poético que se notaba que era de un actor que no estaba formado en un teatro realista. Hacía cosas con el cuerpo que no eran cosas cotidianas, que por ahí tiene más que ver con la formación de los actores populares, ¿no?, pero al mismo tiempo era una manera de contarlo que era muy poética. Y algunas cosas que yo sentía con dificultades en mi formación, y que también las sigo sintiendo, era soltar determinados lugares del cuerpo, poder utilizar el cuerpo para hacer cualquier cosa. No solamente para hacer un personaje realista que le pasan determinadas situaciones. O sea, a mí en lo que tiene que ver con, supongamos agarro una escena de Chéjov o de Tennessee Williams o de Mauricio Kartun, de distintos autores, y tengo que entender cuál es el tránsito emocional del personaje, lo entiendo. Ahora yo tengo que hacer algo que implique un desafío físico más grande, y no tengo el cuerpo acostumbrado para hacerlo. Como esa cosa de mandarse y probar cualquier cosa con el cuerpo y quedar mil veces en ridículo para después ir encontrando cuál es la forma de eso, eso me generaba una dificultad muy grande, ahora todavía, pero un poco menos (Agustina, comunicación personal, 2017)

Agustina empezó a entrenar con Marcelo Savignone; al tiempo él la llamó para trabajar en una obra que dirigía, Mis tres hermanas, una versión de Tres hermanas, de Chéjov. A su vez, terminó la carrera de Artes y empezó 
a trabajar en el grupo de investigación de historia del circo en la Universidad de Tres de Febrero (UNTREF), donde empezó a interesarse por algunas cuestiones ligadas a la tradición del actor popular argentino.

Yo: ¿Sentiste algo de tu mirada que haya cambiado al estudiar con Savignone? Agustina: Empecé a valorar mucho más. Por ejemplo, le empecé a prestar mucha más atención a los tiempos rítmicos de las escenas. De pensar una obra de teatro no solamente como una obra de teatro sino también en algún punto conectarla con el resto de las artes: también es un poco una coreografía y también es un poco una obra musical. Lo cual no tiene que ir en contra de la naturalidad. No es que tenés que decir el texto sí o sí en determinado ritmo porque si no te corrés del tempo, no es lo mismo que una canción. Pero, hay un ritmo de la obra, hay una respiración de la obra, hay una coreografía de la obra, y las obras que logran mantener eso, y juegan mucho y muy bien con eso, y con lo visual, con las composiciones visuales, me parece que tienen un mérito, aunque después uno vea el trabajo del actor y no esté tan bueno como uno querría. Yo siempre prefiero obras donde los actores actúen bien y después venga todo lo demás, si está todo lo demás y el actor no puede sostener lo que está haciendo, me embolo. Y después, algo que empecé a rescatar mucho más, que me parece que tiene que ver con mi incursión en el mundo del circo, más por el lado de la teoría, pero igual, en el mundo del circo, por ese lado, y por el estudio más desde lo físico, y de la técnica Lecoq que es lo que labura Savignone, y empezar a meterme más por ese lado, empecé a meterme y a admirar mucho más las técnicas del actor popular o del actor que labura desde el cuerpo, que no necesariamente por laburar desde el cuerpo sea menos real lo que le pasa, porque ese es un entrenamiento que, yo siento que lo otro se puede entrenar, [en cambio] el actor que tiene ductilidad física y que sabe por ejemplo hacer malabares, o torcer un ojo y el otro no, o dar vuelta... lo que sea, o decir un texto en el tempo que tiene que ir, o ser cómico, todo un montón de cosas que vienen de laburarlo en ambientes extrateatrales, o por fuera del teatro serio, eso es algo que es difícil de aprender, que lo tenés o no lo tenés, o lo laburás o no lo laburás, pero empiezo 


\section{Aprendiendo a lo largo de la ciudad...}

a admirar más a la gente que tiene un cierto humor...

Yo: ¿Quién tiene un cierto humor? Agustina: Osqui Guzmán, que puede bailar, puede hacer una parada de manos, todo mientras está diciendo El Bululú, de José María Vilches. O Chamé Buendía, o los actores de Chamé Buendía, Tincho Lups, por ejemplo, que es el nombre artístico del que hace los cuatro personajes de la obra [se refiere a Otelo, dirigida por Chamé Buendía], y vos decís, ¿este de dónde salió? Él construía cuatro cosas diferentes, y tenía la habilidad de pasar de una cosa a la otra prácticamente sin embrague, y uno dice, si tengo que construir dos comportamientos diferentes para una escena, tengo que estar un tiempo construyéndolos, eso es lo que le pasa muchas veces, no sé si a todo el mundo, pero al actor de formación un poco más tradicional, que le pierde el gusto al juego. Entonces dice, $-\mathrm{y}$ pone una voz seria-, para crear dos comportamientos primero tengo que hacer -y vuelve a su voz - todo este comportamiento de jorobado, no sé... y después hacer todo este otro comportamiento. El actor que viene de otro palo, que tiene esta debilidad de que a veces no se compenetra tanto o no le creés tanto, o no tiene tanta verdad en lo que hace, tiene una ductilidad a veces para jugar con el cuerpo que dice, ¿qué hay que hacer?, tres personajes, y dice bueno, pará, y se va atrás y juega un detalle de cada uno, y vuelve con una propuesta, y no le pierde el disfrute a eso. Y no empieza con... oh, tengo que hacerlo bien y cómo... (Agustina, comunicación personal, 2017).

Agustina empezó a desarrollar una síntesis propia entre su formación con Alezzo, su entrenamiento reciente con Savignone, y otras cuestiones ligadas al actor popular, que empezaron a interesarle a partir de sus investigaciones en la UNTREF, y de la cual ella rescata algunos aspectos ligados a la ductilidad física. Una ductilidad que nota que ella misma, así como otros actores de formación "más tradicional", es decir formados en estilos de actuación cercanos a Stanislavski, no tienen en la misma medida, y que le gustaría desarrollar.

A diferencia de otros casos en los que se da una conversión de un estilo a 
otro y el estudiante suele renegar de su formación anterior, y que no describo aquí por falta de espacio, Agustina no reniega de su formación en el estilo de actuación stanislavskiano. Su formación presente con Savignone la ve como un complemento de algunos elementos que sentía le faltaban, antes que como una nueva comprensión del teatro que anula o minimiza la anterior.

\section{Conclusión:}

La temporalidad de la circulación Al cruzar los estudios de juventud con una aproximación centrada en lo urbano y la ciudad, las investigaciones de Magnani $(2002,2006)$ lograron mostrar cómo los jóvenes recorren, se apropian y se encuentran en la ciudad. Si sus investigaciones han puesto el énfasis en la espacialidad de la circulación, el caso de la formación de los estudiantes de teatro, entendida como una carrera secuencial, nos permite iluminar una serie de aspectos ligados a su temporalidad. Así, vemos que los estudiantes circulan de diferentes maneras en distintos momentos de su formación -lo que es particularmente notorio entre el primer y el tercer momento de la carrera que hemos descrito-. Si en el primer momento la circulación entre maestros está relacionada con criterios territoriales como la cercanía, o en la inserción en otras instituciones como la escuela o centros culturales que el curioso ya frecuentaba antes de hacer teatro, en el tercer momento, la circulación está orientada por formarse al interior de un linaje particular. Así, la circulación queda circunscrita a un circuito organizado en torno a un linaje, lo que no quita que, como mencioné, puedan existir casos de conversión, de un estilo a otro.

También el segundo y el tercer momento son diferentes: si en el segundo momento los estudiantes tienden a ser cautivados por un maestro periférico del linaje, en el tercer momento tienden a circular entre los maestros centrales de este. Por lo tanto, prestar atención a la temporalidad de la circulación también nos permite dar cuenta de ciertas tendencias en el modo en que se circula al interior de un circuito, en diferentes momentos. A su vez, el momento de ser cautivado por un maestro también tiene el interés de llamarnos la atención 


\section{Aprendiendo a lo largo de la ciudad...}

sobre los momentos en que no se circula, es decir, en que la persona decide permanecer más tiempo en un mismo lugar -por lo menos en lo que respecta a su formación-, así como mostrarnos un modo particular en que las personas llegan a circular de una determinada manera o, dicho en otros términos, cómo se inician en un circuito. En este último aspecto, a la cuestión de la temporalidad de la circulación parece agregarse una pregunta por el ritmo de esta (que en unos momentos se acelera -en el primer o el tercer momento-y en otros se detiene -en el segundo-).

Notas

1. El número arrojado por Alternativa Teatral tampoco proviene de un relevamiento estadístico, sino de los "espacios" que decidieron promocionar su show en este sitio principal sitio de promulgación de obras del circuito "off" o alternativo de la Ciudad de Buenos Aires, y muy popularizado entre personas que hacían teatro al momento de mi trabajo de campo.

2. Este trabajo se basa en una investigación etnográfica realizada entre 2014 y 2017. Todas las historias de actores que se mencionan a lo largo de este artículo provienen de entrevistas realizadas entre 2016 y 2017.

3. Utilizo el término estilo en el sentido de Kaeppler, como "patrones persistentes que se presentan en forma de estructuras de representación -que van desde sutiles cantidades de energía al uso de diferentes partes del cuerpoacto reconocido por las personas que pertenecen a una tradición dancística específica" (2003:103). Con respecto al término linaje, lo entiendo como la cadena de maestros y alumnos -algunos de los cuales luego devienen maestros- a través de la cual se enseña una cierta forma de mirar, gozar, pensar, sentir, hacer y querer hacer teatro. Sigo en cierta medida a Rahaim (2012) a quien retomaré más adelante.

4. Diferencio entre circuito realista y estilo de actuación stanislavskiano ya que el circuito realista no comprende a todos los maestros que enseñan en un estilo stanislavskiano en Buenos Aires. El circuito realista solo comprende a los maestros que han aprendido el estilo stanislavskiano con maestros que pertenecen al linaje iniciado por Hedy Crilla en Buenos Aires, cuyos principales representantes fueron Alezzo, Fernandes y Gandolfo (los tres 
principales y más conocidos alumnos de Hedy Crilla). Lo mismo no ocurre para el caso del estilo de actuación del teatro de estados.

5. Esto no quita que algunos estudiantes consideran que ciertos maestros son demasiado violentos en su forma de dirigirse a los estudiantes, y prefieren no estudiar o dejar de estudiar con ellos.

6. La relación entre migración e ir a estudiar con el maestro de su maestro también fue apuntada por Downey (2010) y Dalidowicz (2012) - ver también Downey, Dalidowicz y Mason (2015) - para los casos del aprendizaje del capoeira y la danza kathak, quienes, al decidir continuar su trabajo de campo etnográfico en un país diferente del que lo habían comenzado, cuentan que fueron a estudiar con los maestros de sus maestros -en el caso de Downey en Nueva York y en el de Dalidowicz en Calcuta.

7. Entiendo conversión siguiendo a Berger y Luckman (1972) del siguiente modo. "[E]n la re-socialización [una de cuyas formas es la conversión religiosa] el pasado se re-interpreta conforme con la realidad presente, con tendencia a retroyectar diversos elementos que, en aquel entonces, no estaban subjetivamente disponibles. En la socialización secundaria en cambio el presente se interpreta de modo que se halle en relación continua con el pasado, con tendencia a minimizar aquellas transformaciones que se hayan efectuado realmente.” (Berger y Luckman, 1972, citado por Carozzi y Frigerio, 1994, la aclaración entre corchetes es de los autores). La aplicación del término conversión en este contexto se justifica porque el pasado a menudo se reinterpreta en el sentido de minimizar la influencia de los maestros en el estilo de actuación anterior. Sin embargo, aquí la conversión no implica una redefinición radical de la identidad subjetiva como sugiere la definición de los autores.

8. Durante los años ochentas en Buenos Aires, con la proliferación de nuevos estilos de actuación, este tipo de conversiones parecen haber sido moneda corriente. Tales fueron los casos de actores como Guillermo Angelleli, Claudio Gallardou, Batato Barea y Cristina Martí, que luego de haberse formado entre maestros del circuito realista se pasaron al clown luego de estudiar con Cristina Moreira. 


\section{Referencias}

Battezzati, S. (2017), "Histriónicos y emocionales: dos formas de composición en el teatro de Buenos Aires", Estudios de Teoría Literaria, (6) 11, 95-106.

Becker, H. (2012) Outsiders. Hacia una sociología de la desviación. Buenos Aires: Siglo XXI Editores.

Berger, P. y Luckman T. (1972) La Construcción Social de la Realidad. Buenos Aires: Amorrortu.

Carozzi, M. J. (2000). Nueva Era y terapias alternativas. Construyendo significados en el discurso y la interacción. Buenos Aires:EDUCA.

Carozzi, M. J. y Frigerio A. (1994) Los estudios de la conversión a nuevos movimientos religiosos: perspectivas, métodos y hallazgos, en El estudio científico de la religión a fines del siglo XX. Buenos Aires: CEAL.

Dalidowicz, M. (2012) Learning Kathak: Crafting bodies and selves in the guru shishya parampara. [Ph. D. Thesis]. Department of Anthropology, Macquarie University.

Downey, G. (2010) Practice without theory: a Neuroanthropological Perspective on Embodied Learning, Journal of the Royal Anthropological Institute, 16(1), 22- 40.

Downey, G., Dalidowicz, M. \& Mason, P.H. (2015) Apprenticeship as method: embodied learning in ethnographic practice, Qualitative Research, 15(2), 183- 200.

Frigerio, A. (2012) "Lógicas y límites de la apropiación new age: donde se detiene el sincretismo", en R. de la Torre, C. G. Zúñiga y N. Juárez Huet (comp.), Variaciones y Apropiaciones Latinoamericanas del New Age. México: Centro de Investigaciones y Estudios Superiores en Antropología Social: El Colegio De Jalisco. 
Kaeppler, A. L. (2003) La danza y el concepto de estilo. Desacatos,(12), 93-104.

Magnani, J. G. (1999), O Circuito Neo-esotérico na Cidade de São Paulo en M. J. Carozzi (ed.), A Nova Era no Mercosul. Petrópolis: Vozes [pp. 27-46].

- (2002) De perto e de dentro: notas para uma etnografia urbana, Revista Brasileira de Ciencias Sociais, 17(49).

- (2006), Urban youth circuits in São Paulo, Tempo social, 2(novsept). del Mármol, M. (2016). Una corporalidad expandida. Cuerpo y afectividad en la formación de los actores y actrices en el circuito teatral independiente de la ciudad de La Plata. [Tesis Doctoral]. Universidad de Buenos Aires, Facultad de Filosofía y Letras, Buenos Aires.

Neuman, D. A. (2004) A House of Music: The Hindustani Musician and the Crafting of Traditions. [PhD diss.]. Columbia University.

Osswald, D. (2015) Deshacer los hábitos bailando. Concepciones alternativas del cuerpo en la transmisión dancística independiente en Buenos Aires, en M. J. Carozzi (coord.), Escribir las Danzas. Coreografias de las ciencias Sociales. La Plata: Gorla.

Rahaim M. (2008) "Gesture and Melody in Indian Vocal Music", en Gesture $8(3): 325-347$.

Rahaim M. (2012) Musicking Bodies: Gesture and Voice in Hinduistani music, Middletown: Wesleyan University Press.

Saez, M. (2017), Presencias, riesgos e intensidades. Un abordaje socio- antropológico sobre y desde el cuerpo en los procesos de formación de acróbatas y bailarines/as de danza contemporánea en la ciudad de La Plata. [Tesis de doctorado]. Facultad de Filosofía y Letras, Universidad de Buenos Aires. 


\section{¿Cómo citar este artículo?}

Battezzati, S. (Julio-diciembre, 2018). Aprendiendo a lo largo de la ciudad: la carrera de los estudiantes de actuación en Buenos Aires. Revista humanidades, 8(2), 1-33. doi: http://dx.doi.org/10.15517/h.v\%vi\%i.32470 